\title{
Low-template DNA: a single DNA analysis or two replicates?
}

\section{Abstract}

This study investigates the following two questions: (1) Should the DNA analyst concentrate the DNA extract into a single amplification or should he/she split it up to do two replicates? (2) Given the electropherogram obtained from a first analysis, is it worthwhile for the DNA analyst to invest in obtaining a second replicate? A decision-theoretic approach addresses these questions by quantitatively expressing the expected net gain (ENG) of each DNA analysis of interest. The results indicate that two replicates generally have a greater ENG than a single DNA analysis for DNA quantities capable of producing two replicates having an average allelic peak height as low as 43rfu. This supports the position that two replicates increase the information content with regard to a single analysis.

Keywords: LT-DNA, Replication, Value of information, Decision theory

\section{1. Introduction}

\subsection{Background}

Interpreting low-template DNA (lt-DNA) typing results is challenging because of the increased influence of stochastic effects [e.g., 1]. These stochastic effects can produce a configuration of peaks in the electropherogram (EPG) that is different from the genotype of the DNA's donor. We speak of allele drop-out (or locus drop-out) when one (or both) of the donor's alleles fail(s) to produce a signal in the EPG for a particular locus, and of allele drop-in when a signal for an allele not present in the donor's genotype appears in the EPG. Probabilistic models [e.g., 2] can take the possibility of these stochastic effects into account when assigning the weight of the evidence by modeling the probabilities of allele drop-out [e.g., 3] and allele drop-in [e.g., 4].

To deal with the uncertainty produced by the stochastic effects, a laboratory may perform replicate analyses [1,2]. This involves dividing the DNA into several tubes prior to amplification to produce multiple EPGs (one per tube) for the same sample. The motivation for doing replicates is that the stochastic effects will vary from one replicate to another, so that multiple EPGs may provide more information about the donor's genotype. Benschop et al. [5] and Cowen et al. [6] conclude that replicate analyses combined with a consensus interpretation method (also called biological model) increases the proportion of the donor's genotype that is observed. And according to Steele et al. [7], multiple replicates produce more information than the single profile when a probabilistic interpretation model (also called statistical model) is applied.

Yet, there has been some debate on whether this multiple tube approach provides more information than concentrating the entire DNA sample into a single tube $[8,9,10]$. On the one hand, putting all of the DNA into the same tube may produce an EPG showing fewer allele and locus drop-outs [8]. On the other hand, splitting it into multiple tubes may provide more data for making inferences about the stochastic effects and the donor's genotype [9].

In addition to considering the value of the information produced by each of these two approaches, however, there is also the laboratory's resources to consider. Replicate analyses (i.e., the multiple tube approach) require more resources than performing a single analysis.

This paper investigates the decision of whether it is worth investing in replicates by applying a decision-theoretic approach.

\subsection{Objective}

The objective of this study is to examine the following two questions:

1. all in vs. two replicates: Should the DNA analyst concentrate the DNA extract into a single amplification or should he/she split it up into two tubes to obtain two replicates?

Preprint submitted to Elsevier

March 11, 2016 
2. additional replicate: Given an EPG obtained from a first analysis, is it worthwhile for the DNA analyst to invest in obtaining a second replicate?

The answers to these questions depend on the expected value of information (EVOI) and the laboratory's cost of performing the DNA analyses. Decision theory provides a framework for determining the most rational course of action based on these two values.

This paper is organized as follows: Section 2 describes the methodology of this study, Section 3 presents the results obtained, Section 4 discusses the impact of the various parameters on the results, and Section 5 presents a summary and concluding remarks. The mathematical description of the decision-theoretic model used is in the Appendix.

\section{Materials and methods}

This study consisted of:

1. obtaining DNA profiles for a range of low-level DNA quantities,

2. defining a probabilistic model and its parameters for making inferences on the donor's genotype and on the expected DNA typing results, and

3. defining a decision-theoretic model for quantitatively expressing the EVOI of the DNA typing results.

Points 1 and 2 are described in detail in [11]. This section summarizes points 1 and 2 and describes point 3.

\subsection{DNA analyses}

EPGs of single-source DNA samples were obtained for DNA quantities of 10pg, 7.5pg, 5pg, 2.5pg, $1 \mathrm{pg}, 0.75 \mathrm{pg}$, $0.5 \mathrm{pg}$ and $0.25 \mathrm{pg}$. This range was chosen because it created EPGs ranging from having no allele or locus drop-outs to showing all loci dropping out. ${ }^{1}$

Two kits were used for the DNA amplification: AmpFlSTR $($ Identifiler $($ Plus (29 cycles) and PowerPlexß 16 HS by Promega (32 cycles). Capillary electrophoresis separated and detected the PCR products on am ABI 3130xl genetic sequencer. GeneMapper ID-X software version 1.3 by Applied Biosystems was used for analyzing the DNA typing results. For further details on the analytical procedure, we refer the reader to [11].

\subsection{Probabilistic Model}

A semi-continuous model was used for making inferences on the donor's genotype and on the expected DNA typing results using the equations presented in [2] and the probability assignments listed below:

allele probabilities: point estimates using the allele frequency data presented in [12];

probability of allele drop-out: a logistic regression model for variable $\ln (\hat{H})$ and the parameter values given in [11], where $\hat{H}$ is the average observed peak height in the EPG; and

probability of allele drop-in per locus: values of 0.01 and 0.05 (a set of decision analyses is performed for each value).

We used the $R$ software $^{2}$ to perform the computations. For further details on the probabilistic model, we refer the reader to [11].

\footnotetext{
${ }^{1}$ Analytical threshold of $10 \mathrm{rfu}$

${ }^{2} R$ version 3.1.2, freely available at http://cran.r-project.org/ (last visited April 26, 2015)
} 


\subsection{Decision theoretic model}

The goal of performing DNA analyses is to obtain information about the genotype of the DNA's donor. The analyst has a probability distribution over the donor's possible genotypes, and the information provided by the DNA typing results updates this probability distribution. Yet, alone, this probability distribution does not tell the analyst how to "designate" the donor's genotype, that is, what genotype to assign so that the analyst can compare the DNA typing results with the genotypes of potential donors and exclude the ones that don't fit the designation. This genotype designation process is a decision [13]. The analyst chooses a designation based on the probability distribution over the donor's possible genotypes and the utility function describing the benefit of a correct designation and the cost of an incorrect designation. Making a rational decision requires choosing the designation that maximizes the expected utility [e.g., 14].

For decisions about performing or not performing DNA analyses and what DNA analyses to perform, a rational decision-maker requires the expected net gains (ENGs) of the analyses in question. Let us call the DNA analysis of interest $t^{*}$. The ENG of DNA analysis $t^{*}$ is equal to the EVOI of $t^{*}$ minus the cost of performing $t^{*}$ :

$$
\operatorname{ENG}\left(t^{*}\right)=\operatorname{EVOI}\left(t^{*}\right)-\operatorname{cost}\left(t^{*}\right)
$$

A rational decision-maker will choose the action that maximizes the ENG [15]. If the $\mathrm{ENG}\left(t^{*}\right)$ is negative, performing no DNA analysis is more rational, because the ENG of performing no analysis is equal to zero. The mathematical development for this decision-theoretic model for the DNA analyses of interest in this study is given in the Appendix.

The EVOI of a DNA analysis (Eqs. (A.2) and (A.3) in the Appendix) depends on the probabilistic model (see Section 2.2) and the utility function. We discuss the utility function below.

\subsubsection{Utility function}

There are three types of genotype designations for each locus:

complete: a complete designation that names two alleles, for example $\{7,8\}$;

partial: a designation that specifies only one allele and uses a wildcard $F$ for the other allele, where $F$ represents any allele at this locus, for example $\{7, F\}$; and

uninformative: an uninformative designation $\{F, F\}$, that is, a designation that doesn't specify any allele at this locus.

For each locus, the designation of a genotype can lead to four possible outcomes:

an incorrect designation: The genotype designation is different from the donor's genotype. This difference leads to an incorrect exclusion of the donor's genotype as a possible source of the DNA.

an uninformative designation $\{F, F\}$ : The genotype designation does not say anything about the donor's genotype. All possible genotypes are included as possible genotypes of the DNA's source.

a correct partial designation: The allele in the genotype designation is identical to at least one of the donor's alleles (the other allele designation is a wildcard). This designation leads to correct exclusions of all genotypes that do not contain this allele, yet includes both the donor's genotype as well as genotypes with a one-allele difference to the donor's genotype as possible genotypes of the DNA's source.

a correct complete designation: The genotype designation is identical to the donor's genotype. This designation leads to correct exclusions of all genotypes that are different from the donor's genotype, retaining only the genotype of the DNA's source.

A utility function describes an analyst's preferences among these outcomes. We define a cardinal utility function ${ }^{3}$ which specifies two things:

\footnotetext{
${ }^{3}$ A cardinal utility function measures the utility quantitatively, as opposed to an ordinal utility function which gives only qualitative rankings without any numerical measures [e.g., 16].
} 
1. preference structure: the relative costs and benefits of each of the possible outcomes to each other, for example a correct complete designation is two times more desirable than a correct designation with $F$; and

2. magnitude: the absolute cost or benefit of each outcome, for example the utility of a correct complete designation is 100 utiles $^{4}$.

The preference structure determines what action maximizes the expected utility. In this study, we used two different preference structures, which we call the symmetric preference structure and the conservative preference structure. Rows 1 and 2 in Table 1 presents these on a scale from 0 to 1 . Both of these preference structures assume that the decision-maker is indifferent between the following two hypothetical gambles (i.e., they both assume probabilities of 0.5 and 0.5 in gamble 1 ):

gamble 1: obtain a correct complete designation with a probability of 0.5 and an uninformative designation $\{F, F\}$ with a probability of 0.5

gamble 2: obtain a correct partial designation with certainty

The two preference structures differ in the value they assign to $x$ to make the decision-maker indifferent between the following two gambles (i.e., they assume different probabilities in gamble 1, represented here by $x$ and $1-x$, and the value of $x$ for each is presented below):

gamble 1: obtain a correct complete designation with a probability of $x$ and an incorrect designation with a probability of $1-x$

gamble 2: obtain an uninformative designation $\{F, F\}$ with certainty

For the symmetric preference structure $x=0.5$, and for the conservative preference structure $x=0.9$. The conservative preference structure is more conservative because switching from an uninformative designation to a designation that specifies the alleles requires a higher probability that the allelic designation will be correct. So this preference structure penalizes an incorrect designation more than the symmetric preference structure does. The preference structure encapsulates the decision-maker's risk-taking behavior with regard to the risk of a false exclusion and the risk of a false inclusion. Lowering one risk increases the other. So the most acceptable preference structure is the most desirable balance between these two risks, which should be based on the economic and social costs involved [18]. In this case, the conservative preference structure leads to a smaller probability of a false exclusion and a larger probability of false inclusion, and the symmetric preference structure leads to a smaller probability of false inclusion and a larger probability of false exclusion. In casework, the severity of the committed crime may determine the preference structure of the forensic scientist's utility function: the more severe the crime is, the more conservative the preference structure because the greater the forensic scientist's desirability of reducing the probability of a false exclusion.

The magnitude of the utility function specifies how large the maximum expected utility is, and therefore how large the EVOI is. To quantitatively express the ENG on a monetary scale, it is necessary to define the magnitude of the utility function on this same monetary scale. In this paper we use US dollars as the monetary scale. We define magnitude $m$ as the gain in dollars of a correct complete designation for one locus. Centering our utility function at 0 for the uninformative designation $\{F, F\}$ (i.e., an uninformative designation produces zero gain and zero loss), this means that a correct partial designation produces a gain of $\frac{m}{2}$ dollars, and an incorrect designation yields a loss of $-m$ dollars in the symmetric preference structure and a loss of $-9 m$ dollars in the conservative preference structure (rows 3 and 4 in Table 1) for one locus. The magnitude of the utility function in a particular case requires considering the monetary costs of false inclusions and false exclusions. This is a difficult concept to quantify. It includes investigational costs of investigating potential donors. The investigational resources a police force can invest for a particular case may, again, depend on the severity of the committed crime. If it can be assumed that the DNA was left by the offender, then $m$ can also include society's costs with regard to letting the offender go free and convicting an innocent person. In this case, $m$ can depend on:

- the probability of reoffending: the higher this probability, the greater the cost of a false exclusion;

${ }^{4} \mathrm{~A}$ utile is a basic unit of measurement of utility [17]. 


\begin{tabular}{lcccc}
\hline & $\begin{array}{c}\text { incorrect } \\
\text { designation }\end{array}$ & $\begin{array}{c}\text { uninformative } \\
\text { designation }\{F, F\}\end{array}$ & $\begin{array}{c}\text { correct partial } \\
\text { designation }\end{array}$ & $\begin{array}{c}\text { correct complete } \\
\text { designation }\end{array}$ \\
\hline scale from 0 to 1 & 0 & 0.5 & 0.75 & 1 \\
symmetric preference structure & 0 & 0.9 & 0.95 & 1 \\
$\begin{array}{l}\text { conservative preference structure } \\
\text { monetary scale }\end{array}$ & 0 & 0 & & \\
symmetric preference structure & $-m$ & 0 & $\frac{m}{2}$ & $m$ \\
conservative preference structure & $-9 m$ & $\frac{m}{2}$ & $m$ \\
\hline
\end{tabular}

Table 1: The symmetric utility function and the conservative utility function shown here on a scale from 0 to 1 (rows 1 and 2 ) and on a monetary scale where the utility of an uninformative designation $\{F, F\}$ is set to 0 and $m$ represents the magnitude.

- the seriousness of the committed crime: the more serious the committed the crime, the greater the cost of a false exclusion (in particular if the probability of reoffending is high); and

- if the DNA is the only piece of evidence and can lead to a conviction, then the expected sentence for the committed crime: the more severe the sentence, the greater the cost of a false inclusion.

To avoid focusing on any one scenario, this study performed the decision analyses for a range of values of $m$, consisting of $1,10,100,1000$ and 10000 .

Note that this utility function assigns a loss or gain for the genotype designation at one locus, and the DNA profiles we are considering consist of 15 loci. So the maximum gain and loss for an entire genotype (i.e., for 15 loci) are equal to 15 times the values presented here.

\subsubsection{Cost of DNA analyses}

Quantitatively expressing the ENG requires specifying the cost of the DNA analysis of interest. To answer the two questions presented in Section 1.2, we need the cost of performing a single DNA analysis for the sample and the cost of performing two replicate analyses for this sample. We define cost $c_{1}$ as the cost in US dollars of a single DNA analysis, and cost $c_{2}$ as the cost in US dollars of two replicate analyses.

If we consider only the cost of the DNA analysis kits, we estimate the cost of one DNA analysis as $\$ 45 .{ }^{5}$ The cost of two replicates would be a maximum of $\$ 90$, but most likely less since the quantification and extraction stages would be performed only once.

If we attempt to consider the total cost of one DNA analysis, that is if we take into account the labor, the equipment, facilities, etc. in addition to the DNA analysis kits, the cost values would be much higher. To see how the results of the decision analysis vary in function of the costs, we repeated the decision analysis for costs of $\$ 450$ for one DNA analysis and $\$ 900$ for two replicates. The cost of $\$ 900$ for two replicates assumes again that performing two replicates uses twice as many resources as one analysis. This is the maximum cost for two replicates. The real cost is most likely smaller. For this reason we repeated this decision analysis for a cost of $\$ 600$ for two replicates (which considers the additional cost of the second replicate to be $\$ 150$, or $\frac{1}{3}$ of the cost of a single analysis).

\subsubsection{All in vs. two replicates}

We obtained numerical values for the ENG of a single DNA analysis of a quantity $q$ of DNA, denoted $E N G\left(t_{1, q}\right)$, and the ENG of two replicate analyses, each for a quantity $\frac{q}{2}$ of DNA, denoted $E N G\left(t_{2, \frac{q}{2}}\right)$. We performed this decision analysis for $q$ of what we quantified as $\sim 10 \mathrm{pg}, \sim 5 \mathrm{pg}, \sim 1 \mathrm{pg}$ and $\sim 0.5 \mathrm{pg}$. For such small quantities of DNA, these quantification values should be taken with caution. So instead of presenting the results in function of the quantity of DNA, we present the results in function of the average observed peak height of an allele in the resulting EPG (Table 2). We compared the ENG of one DNA analysis for each of the average peak heights $q$ with the ENG of two replicates, each with the average peak height of $\frac{q}{2}$. We assessed $E V O I\left(t_{1, q}\right)$ and $E V O I\left(t_{2, \frac{q}{2}}\right)$ in R using Eq. A.2 (in the Appendix).

\footnotetext{
${ }^{5}$ The authors thank Jo Bright and Todd Bille for their valuable insight that helped inform this value.
} 
Identifilerß Plus:

\begin{tabular}{llll}
\hline & all in for 1 analysis $\left(t_{1, q}\right)$ & 2 replicates $\left(t_{2, \frac{q}{2}}\right)$ & comparison of $\operatorname{EVOI}\left(t_{1, q}\right)$ with $\operatorname{EVOI}\left(t_{2, \frac{q}{2}}\right)$ \\
\hline quantity 1 $(\sim 0.5 \mathrm{pg}):$ & $\hat{H}=18 \mathrm{rfu}$ & $\hat{H}=8 \mathrm{rfu}$ & $\mathbf{E V O I}\left(\mathbf{t}_{1,0.5}\right)>\operatorname{EVOI}\left(t_{2,0.25}\right)$ \\
quantity 2 $(\sim 1.0 \mathrm{pg}):$ & $\hat{H}=43 \mathrm{rfu}$ & $\hat{H}=18 \mathrm{rfu}$ & $\mathbf{E V O I}\left(\mathbf{t}_{1, \mathbf{1}}\right)>\operatorname{EVOI}\left(t_{2,0.5}\right)$ \\
quantity 3 $(\sim 5.0 \mathrm{pg}):$ & $\hat{H}=287 \mathrm{rfu}$ & $\hat{H}=114 \mathrm{rfu}$ & $\operatorname{EVOI}\left(t_{1,5}\right)<\operatorname{EVOI}\left(\mathbf{t}_{2,2.5}\right)$ \\
quantity 4 $(\sim 10.0 \mathrm{pg}):$ & $\hat{H}=555 \mathrm{rfu}$ & $\hat{H}=287 \mathrm{rfu}$ & $\operatorname{EVOI}\left(t_{1,10}\right)<\operatorname{EVOI}\left(\mathbf{t}_{2,5}\right)$ \\
\hline
\end{tabular}

PowerPlex® 16 HS:

\begin{tabular}{|c|c|c|c|}
\hline & all in for 1 analysis $\left(t_{1, q}\right)$ & 2 replicates $\left(t_{2, \frac{q}{2}}\right)$ & comparison of $E V O I\left(t_{1, q}\right)$ with $E V O I\left(t_{2, \frac{q}{2}}\right)$ \\
\hline quantity $1(\sim 0.5 \mathrm{pg})$ : & $\hat{H}=43 \mathrm{rfu}$ & $\hat{H}=21 \mathrm{rfu}$ & $\operatorname{EVOI}\left(\mathbf{t}_{1,0.5}\right)>E V O I\left(t_{2,0.25}\right)$ \\
\hline quantity 2 ( $1.0 \mathrm{pg})$ : & $\hat{H}=95 \mathrm{rfu}$ & $\hat{H}=43 \mathrm{rfu}$ & $\begin{array}{l}E V O I\left(t_{1,1}\right)<E V O I\left(t_{2,0.5}\right) \text {, except for a conservative } \\
\text { preference structure with a probability of allele } \\
\text { drop-in of } 0.05 \text { where } \operatorname{EVOI}\left(t_{1,1}\right)>\operatorname{EVOI}\left(t_{2,0.5}\right)\end{array}$ \\
\hline quantity 3 ( 5.0pg): & $\hat{H}=530 \mathrm{rfu}$ & $\hat{H}=194 \mathrm{rfu}$ & $\operatorname{EVOI}\left(t_{1,5.0}\right)<\mathbf{E V O I}\left(\mathbf{t}_{2,2.5}\right)$ \\
\hline quantity $4(\sim 10.0 \mathrm{pg})$ : & $\hat{H}=772 \mathrm{rfu}$ & $\hat{H}=530 \mathrm{rfu}$ & $\operatorname{EVOI}\left(t_{1,10}\right)<\operatorname{EVOI}\left(\mathbf{t}_{2,5}\right)$ \\
\hline
\end{tabular}

Table 2: Mean average peak heights in an EPG for the quantities of DNA $(q)$ of approximately $0.5 \mathrm{pg}, 1 \mathrm{pg}, 5 \mathrm{pg}$ and $10 \mathrm{pg}$ used in the all in vs. two replicates study and comparison of the EVOI of a single DNA analysis, EVOI $\left(t_{1, q}\right)$, with the EVOI of two replicates, $E V O I\left(t_{2, \frac{q}{2}}\right)$, for each of these quantities. The larger EVOI is highlighted in bold if it is larger for all situations represented in that row.

\subsubsection{Additional replicate}

We obtained numerical values for the ENG of a second replicate given the DNA typing results of a first DNA analysis, denoted $E N G\left(t_{+1}\right)$. We performed this decision analysis for the entire range of the DNA dilution series (see Section 2.1 and Section 2.1.1. in [11]), assuming that the second replicate would have the same expected average allelic peak height as the first analysis. We assessed $E V O I\left(t_{+1}\right)$ in R using Eq. A.3 (in the Appendix).

\section{Results}

\subsection{All in vs. two replicates}

We note that the average peak heights are about twice as large for the PowerPlex $₫ 16$ HS results than for the Identifiler $\AA$ Plus results (Table 2). This is a consequence of the difference in the manufacturer's recommended amplification cycle number (i.e., 29 cycles for Identifiler ${ }^{\circledR}$ Plus and 32 cycles for PowerPlex ${ }^{\circledR} 16$ HS). These mean average peak heights determined the probability of allele drop-out (see Eq. (1) in [11]) used in the probabilistic part of the model.

Comparing the EVOI obtained for a single analysis with the EVOI obtained for two replicates for each of the four quantities of DNA produced the following results (Table 2):

Identifiler@ Plus: The EVOI of a single analysis is greater than the EVOI of two replicates for quantities 1 and 2, and smaller than the EVOI of two replicates for quantities 3 and 4.

PowerPlex@ 16 HS: The EVOI of a single analysis is greater than the EVOI of two replicates for quantity 1, and smaller than the EVOI of two replicates for quantities 3 and 4. For quantity 2, the EVOI of a single analysis is smaller than the EVOI of two replicates for all of the scenarios studied except for the scenario of a conservative preference structure with a probability of allele drop-in of 0.05 . In this scenario, the EVOI of a single analysis is greater than the EVOI of two replicates.

Examining these results with regard to the mean average allelic peak heights for each of the DNA quantities leads to the following observations. The EVOI of a single analysis is greater than the EVOI of two replicates when the average peak height in an EPG of a single analysis is 43rfu or less (this corresponds to the average peak height in each of two replicates being about 20rfu or less). The EVOI of two replicates is greater than the EVOI of a single analysis 
when the average peak height in an EPG of a single analysis is $287 \mathrm{rfu}$ or more (this corresponds to the average peak height in each of two replicates being about 114rfu or more). The threshold where the EVOI of two replicates becomes greater than the EVOI of a single analysis lies around an average peak height in an EPG of a single analysis of about $95 \mathrm{rfu}$ (this corresponds to the average peak height in each of two replicates being around 43rfu). At this threshold, the utility function's preference structure and the value of the probability of allele drop-in determine which analysis has the greater EVOI.

Combining the EVOI of the DNA analyses with their costs produces the ENGs presented in Figs. 1 through 8 in [11]. Figures 1 through 4 present the Identifiler@ Plus results: Figs. 1 and 2 are for a symmetric preference structure and Figs. 3 and 4 for a conservative preference structure, and Figs. 1 and 3 are for a probability of allele drop-in of 0.01 and Figs. 2 and 4 for a probability of allele drop-in of 0.05 . Figures 5 through 8 present the PowerPlex 16 HS results: Figs. 5 and 6 are for a symmetric preference structure and Figs. 7 and 8 for a conservative preference structure, and Figs. 5 and 7 are for a probability of allele drop-in of 0.01 and Figs. 6 and 8 for a probability of allele drop-in of 0.05 . In each figure, the utility function's magnitude $m$ increases from left to right, and each row presents the results for different DNA analysis cost values $c_{1}$ and $c_{2}$.

When $m$ is two orders of magnitude smaller than $c_{1}$ and $c_{2}$, the ENG of both a single analysis and two replicates are negative. This indicates that the most rational choice is not to perform any DNA analysis.

As $m$ increases with regard to $c_{1}$ and $c_{2}$, the EVOIs of one and two replicates become greater than their costs, and the EVOI of the DNA analyses become dominant in determining the most rational choice. Hence, we find that the most rational choices for the situations where $m$ is at least the same order of magnitude as $c_{1}$ and $c_{2}$ correspond to the DNA analyses with the greater EVOI.

Going from a symmetric preference structure to a conservative preference structure increases the threshold value of the average allelic peak height where performing two replicates becomes more rational than a single analysis.

Increasing the probability of allele drop-in from 0.01 to 0.05 has a negligible effect for Identifiler $®$ Plus and causes a slight increase of the threshold average allelic peak height value for PowerPlex $\AA 16$ HS.

\subsection{Additional replicate}

Figures 9 through 16 in [11] present the ENGs of an additional replicate in function of the average allelic peak height of the first DNA analysis's EPG. Figures 9 through 12 present the Identifiler® Plus results: Figs. 9 and 10 are for a symmetric preference structure and Figs. 11 and 12 for a conservative preference structure, and Figs. 9 and 11 are for a probability of allele drop-in of 0.01 and Figs. 10 and 12 for a probability of allele drop-in of 0.05 . Figures 13 through 16 present the PowerPlex $₫$ 16 HS results: Figs. 13 and 14 are for a symmetric preference structure and Figs. 15 and 16 for a conservative preference structure, and Figs. 13 and 15 are for a probability of allele drop-in of 0.01 and Figs. 14 and 16 for a probability of allele drop-in of 0.05. In each figure, the utility function's magnitude $m$ increases from left to right, and each row presents the results for different DNA analysis cost values $c_{1}$. Figure 1 reproduces here the graph showing the Identifiler ${ }^{\circledR}$ Plus results for a probability of drop-in of $0.01, c_{1}=\$ 45$, and a utility function with a symmetric preference structure and $m=100$.

The graphs in these figures show a concave increase of the ENG with regard to the average allelic peak height. For the DNA samples quantified at about $\sim 0.25 \mathrm{pg}$ to $\sim 2.5 \mathrm{pg}$, the ENG of an additional replicate decreases as the average allelic peak height of the first DNA analysis's EPG increases. So for these quantities, the ENG is at its greatest when the first amplification fails completely, because that's when the second replicate can produce the largest amount of additional information. For all DNA samples quantified at about $\sim 5 \mathrm{pg}$ and greater, there is an expectation of obtaining a complete profile. This causes the ENG of a second replicate to stop increasing in function of the DNA's quantitation and plateau in the graphs.

An ENG greater than 0 indicates that it is rational to do a second replicate. With regard to this threshold, the graphs in these figures fall into three categories.

1. $m$ is at least one order of magnitude smaller than $c_{1}$ : The cost of an additional replicate is always greater than the EVOI of the additional DNA typing results, producing a negative ENG. In this case, it is not rational to do a second replicate.

2. $m$ is the same order of magnitude as $c_{1}$ : The cost of an additional replicate is greater than the EVOI of the additional replicate for small quantities of DNA, and smaller than the EVOI of the additional replicate for larger quantities of DNA. The threshold between these two situations is marked by the horizontal dashed line at 0 
Figure 1: The Identifiler@ Plus results of the additional replicate study for a probability of allele drop-in of 0.01 , a cost $c_{1}$ of $\$ 45$ per DNA analysis, and a utility function with a symmetric preference structure and a magnitude $m$ of 100 . The graph plots the ENGs of a second replicate $E N G\left(t_{+1}\right)$ in function of the average allelic peak height (in rfu) of the first DNA analysis's EPG for DNA samples quantified as $\sim 0.25 \mathrm{pg}$ (red), $\sim 0.5 \mathrm{pg}$ (orange), $\sim 0.75 \mathrm{pg}$ (yellow), 1 pg (green), 2.5pg (turquoise), $\sim 5 \mathrm{pg}$ (blue), 7.5pg (light magenta), and $\sim 10 \mathrm{pg}$ (dark magenta).

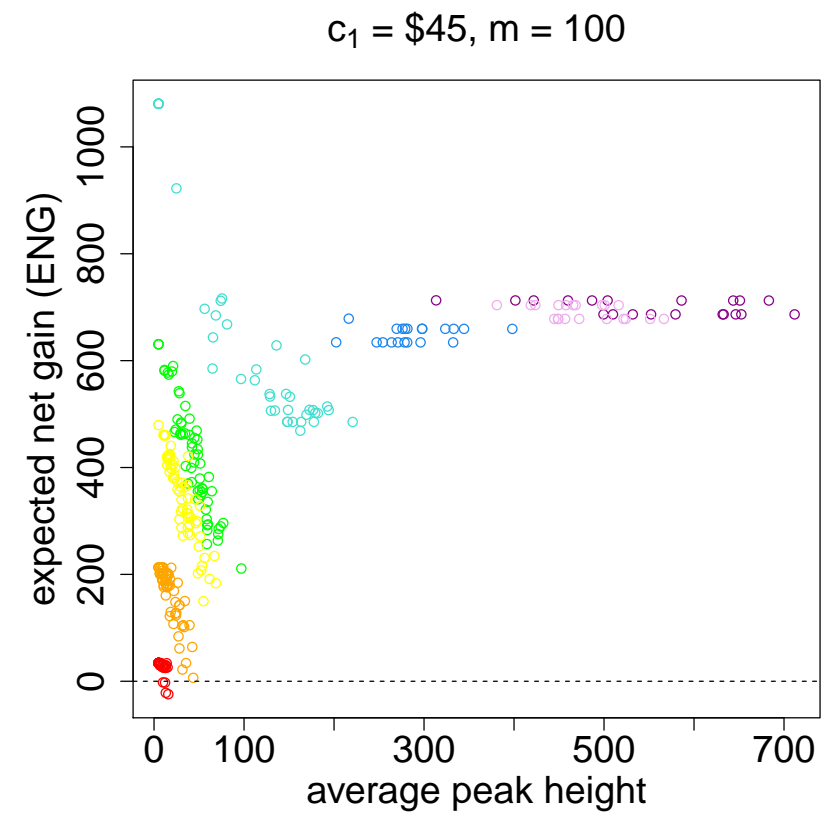

in these graphs. This threshold is at quantities of DNA having a mean average peak height of 43rfu. In the situation where the utility function has a conservative preference structure and the probability of allele drop-in is 0.05 , the ENG is positive for the majority (Identifiler@ Plus) or all (PowerPlex $\AA 16$ HS) analyzed samples of the threshold quantity. In this situation, it seems to be rational to do a second replicate whenever the DNA quantity falls into a category having a mean average allelic peak height of $\geq 43 \mathrm{rfu}$. For all other situations, the ENG can be positive or negative for the analyzed samples of the threshold quantity. In these situations, it is rational to do a second replicate if the DNA quantity falls into a category having a higher mean average allelic peak height than the threshold quantity (e.g., $\geq 114$ rfu for Identifiler@ Plus and $\geq 82$ rfu for PowerPlex@ 16 HS).

3. $m$ is at least one order of magnitude greater than $c_{1}$ : In the majority of the cases, the EVOI of the additional replicate is greater than the cost of a second replicate, producing a positive ENG. In these cases, it is rational

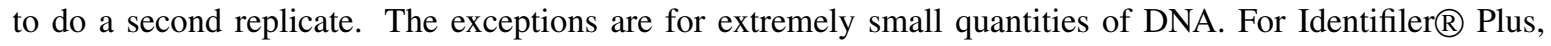
these are quantities where the mean average peak height is $\leq 18 \mathrm{rfu}$ when the utility function has a conservative preference structure, and quantities where the mean average peak height is $\leq 8 \mathrm{rfu}$ when the utility function has a symmetric preference structure. For PowerPlex $₫ 16$ HS, the exceptions are quantities where the mean average peak height is $\leq 21 \mathrm{rfu}$ when the utility function has a conservative preference structure and $m$ is one order of magnitude greater than $c_{1}$. In these cases, the ENG can be negative, so that it is not rational to do a second replicate.

Going from a symmetric preference structure to a conservative preference structure lowers the threshold for category 2 when the probability of allele drop-in is 0.05 , and causes a very slight increase of the threshold for category 3 . Increasing the probability of allele drop-in from 0.01 to 0.05 lowers the threshold for the scenarios in category 2 when the preference structure is conservative. 


\section{Discussion}

First we note that it is not worth performing any DNA analysis when $m$ is more than one order of magnitude smaller than the cost. Such small values of $m$ represent low costs of false inclusions and false exclusions. This occurs in cases of extremely minor offenses and in cases where there is a large uncertainty on the relevance of the DNA. The EVOI of the DNA analyses becomes overpowered by their costs, so that a decision analysis favors the choice with the smallest cost: not perform any DNA analysis.

For more serious cases with relevant biological samples, the model's results correspond with the expectation that two replicates produce more information than a single analysis. This result holds down to very small quantities of DNA. The threshold where the optimal choice switches from two replicates to a single analysis is around the point where the average allelic peak height in a replicate is $43 \mathrm{rfu}$, if the laboratory uses an analytical threshold between $10 \mathrm{rfu}$ and 50rfu.

In the additional replicate study, the threshold drops to 20rfu or less when $m$ is at least one order of magnitude greater than the cost. In these cases, the costs of false inclusions and false exclusions are much greater than the cost of a DNA analysis, so that it is worthwhile investing in an additional analysis even when the expectation for gaining additional information is very low. This occurs for high profile cases, where it is worthwhile obtaining as much information as possible regardless of the financial cost.

Sensitivity analyses of the utility function's preference structure and the probability of allele drop-in show minor fluctuations of the threshold value. A greater penalty for false designations (achieved by making the utility function's preference structure more conservative) gives the information content of the results of very small quantities of DNA less value because more information is needed to make informative genotype designations to reduce the risk of false genotype designations. Increasing the probability of allele drop-in increases the uncertainty on the donor's genotype for very small quantities of DNA, and this lowers the information content of the results. Hence a more conservative utility function and a higher probability of allele drop-in lower the EVOI of two replicates for very small quantities of DNA. This reduction in the EVOI of two replicates shifts the optimal choice towards a single analysis. In the all in vs. two replicates study, making the preference structure more conservative and raising the probability of allele drop-in therefore raises the threshold value of the minimum average allelic peak height in each of the replicates. For the additional replicate study, we distinguish between situations where $m$ is the same order of magnitude as the cost (the threshold is around 43rfu) and situations where $m$ 's order of magnitude is greater than the cost (the threshold is around 20rfu or lower). In the first case, the decrease of the value of the information of the results of the first DNA analysis makes the information content of an additional replicate more valuable (i.e., increases the EVOI of an additional replicate), and hence slightly lowers the threshold. In the second case, the EVOI of the additional replicate decreases for very small quantities of DNA so that the cost of the DNA analysis no longer counterbalances its EVOI, and this slightly raises the threshold. With regard to the parameter values examined in this study (i.e., the symmetric vs. the conservative preference structure, and a probability of allele drop-in of 0.01 vs. 0 . per locus), it is the utility function's preference structure that had the greater impact. It is possible that a larger probability of allele drop-in might have a larger impact than the results observed in this study, yet according to the values reported in the literature [e.g., 1, 3, 19, 20], it is not reasonable to assume a probability of allele drop-in greater than 0.05 per locus.

The logistic regression curves for the two different kits were similar enough that there was no perceivable difference between the two amplification kits.

\section{Conclusions}

The results of this study indicate that two replicates generally have a greater ENG than a single DNA analysis for DNA quantities producing two replicates that each have an average allelic peak height of as low as 43rfu. With regard to the all in vs. two replicates question, this means that the DNA analyst should split the DNA extract into two tubes to obtain two replicates when there is enough DNA that the expected allelic peak height in each replicate is at least 43rfu. With regard to the additional replicate question, this means that it is worthwhile for the DNA analyst to invest in obtaining a second replicate when the expected average allelic peak height is greater than 43rfu. This value of $43 \mathrm{rfu}$ should be taken as a general indication that applies to many of the scenarios examined in this study, and not as an absolute threshold value. Sensitivity analyses show that the threshold value depends to a large extent on the 
magnitude of the utility function, to a small extent on the preference structure of the utility function, and to a very small extent on the assigned probability of allele drop-in.

The target quantity of DNA for lt-DNA analyses in casework is 500pg [21, 22]. The results of this study present the limits of this method for quantities between 0.25 and 10pg (i.e., quantities that are 50 to 2000 times smaller than the target quantity). Hence, the threshold where a single DNA analysis becomes more rational than two replicates is at quantities of 2 to 3 orders of magnitude smaller than the target amount for lt-DNA.

The results of this study support Kokshoorn and Blankers [9] and Steele et al. [7] in that two replicates increase the information content with regard to a single DNA analysis, and thus provide more data for making inferences about the donor's genotype.

\section{Appendix A. Decision-theoretic model}

We denote the donor's possible genotypes $\boldsymbol{\theta}_{\mathbf{1}}, \boldsymbol{\theta}_{\mathbf{2}}, \ldots, \boldsymbol{\theta}_{\boldsymbol{n}}$. Each $\boldsymbol{\theta}_{\boldsymbol{j}}, j=1, \ldots, n$, is a vector describing the donor's genotype for 15 loci:

$$
\boldsymbol{\theta}_{j}=\left\{\theta_{j 1}, \theta_{j 2}, \ldots, \theta_{j 15}\right\} .
$$

Next we denote the possible designations of the donor's genotype $\boldsymbol{a}_{\mathbf{1}}, \boldsymbol{a}_{2}, \ldots, \boldsymbol{a}_{\boldsymbol{m}}$. Each $\boldsymbol{a}_{\boldsymbol{i}}, i=1, \ldots, m$, is a vector describing the genotype designation for 15 loci:

$$
\boldsymbol{a}_{i}=\left\{a_{i 1}, a_{i 2}, \ldots, a_{i 15}\right\} .
$$

The consequence $C\left(a_{i l}, \theta_{j l}\right), l \in\{1,2, \ldots, 15\}$, is the consequence of the decision-maker choosing designation $a_{i l}$, $i \in\{1,2, \ldots, m\}$, when $\theta_{j l}, j \in\{1,2, \ldots, n\}$, is true. We will abbreviate $C\left(a_{i l}, \theta_{j l}\right)$ by $C_{i j l}$. The utility value assigned to consequence $C_{i j l}$ is denoted $u\left(C_{i j l}\right)$.

Without observing any DNA typing results, the maximum expected utility is:

$$
\max _{i} \bar{u}\left(\boldsymbol{a}_{\boldsymbol{i}} \mid I\right)=\sum_{l=1}^{15} \max _{i} \sum_{j=1}^{n} u\left(C_{i j l}\right) \operatorname{Pr}\left(\theta_{j l} \mid I\right),
$$

where the $I$ after the conditioning bar represents the background information that informs the probability distribution over the donor's possible genotypes (e.g., what kit was used and information on allele frequencies in the relevant population). We denote the possible results of a particular DNA analysis $t^{*}$ as $\boldsymbol{e}_{1}^{t^{*}}, \boldsymbol{e}_{2}^{t^{*}}, \ldots, \boldsymbol{e}_{s}^{t^{*}}$, where each $\boldsymbol{e}_{k}^{t^{*}}, k=$ $1, \ldots, s$, is a vector describing the observed allelic peaks for 15 loci:

$$
\boldsymbol{e}_{\boldsymbol{k}}^{t^{*}}=\left\{e_{k 1}^{t^{*}}, e_{k 2}^{t^{*}}, \ldots, e_{k 15}^{t^{*}}\right\}
$$

The expected value of information of DNA analysis $t^{*}$, denoted $E V O I\left(t^{*}\right)$, is the difference between the expected maximum utility with the results of this DNA analysis and the expected maximum utility without these results:

$$
\operatorname{EVOI}\left(t^{*}\right)=\sum_{l=1}^{15} \sum_{k=1}^{s} \max _{i} \bar{u}\left(a_{i l} \mid e_{k l}^{t^{*}}, I\right) \operatorname{Pr}\left(e_{k l}^{t^{*}} \mid I\right)-\max _{i} \bar{u}\left(\boldsymbol{a}_{i} \mid I\right) .
$$

We used this equation to obtain $\operatorname{EVOI}\left(t_{1, q}\right)$ and $\operatorname{EVOI}\left(t_{2, \frac{q}{2}}\right)$, the EVOIs necessary for quantitatively expressing $E N G\left(t_{1, q}\right)$ and $E N G\left(t_{2, \frac{q}{2}}\right)$ to address the "all in vs. multiple replicates" question (Sections 2.3.3 and 3.1).

The EVOI necessary for addressing the "additional replicate" question, denoted $E V O I\left(t_{+1}\right)$, is the difference between the expected maximum utility with the DNA results of the additional replicate and expected maximum utility without these results. The following equation gives the EVOI of a second replicate given the results of the first analysis:

$$
\operatorname{EVOI}\left(t_{+1}\right)=\sum_{l=1}^{15} \sum_{k=1}^{s} \max _{i} \bar{u}\left(a_{i l} \mid e_{k l}^{t_{2}}, I\right) \operatorname{Pr}\left(e_{k l}^{t_{2}} \mid I\right)-\max _{i}, \bar{u}\left(\boldsymbol{a}_{i} \mid \boldsymbol{e}_{*}^{t_{1}}, I\right)
$$

where $\boldsymbol{e}_{*}^{\boldsymbol{t}_{1}}$ represents the DNA typing results obtained from the first DNA analysis (i.e., replicate 1). We used this equation for $\operatorname{EVOI}\left(t_{+1, q}\right)$, the EVOI necessary for quantitatively expressing $E N G\left(t_{+1, q}\right)$ to address the "additional replicate" question (Sections 2.3.4 and 3.2). 


\section{References}

[1] P. Taberlet, S. Griffin, B. Goossens, S. Questiau, V. Manceau, N. Escaravage, L.P. Waits, J. Bouvet, Reliable genotyping of samples with very low DNA quantities using PCR, Nucleic Acids Research 24 (1996) 3189-3194.

[2] P. Gill, J.P. Whitaker, C. Flaxman, N. Brown, J. Buckleton, An investigation of the rigor of interpretation rules for STRs derived from less than 100 pg of DNA, Forensic Sci. Int. 112 (2000) 17-40.

[3] P. Gill, A. Kirkham, J.M. Curran, LoComatioN: A software tool for the analysis of low copy number DNA profiles, Forensic Sci. Int. 166 (2007) 128-138.

[4] R. Puch-Solis, A dropin peak height model, Forensic Sci. Int.: Genet. 11 (2014) 80-84.

[5] C.C.G. Benschop, C.P. van der Beek, H.C. Meiland, A.G.M. van Gorp, A.A. Westen, T. Sijen, Low template STR typing: Effect of replicate number and consensus method on genotyping reliability and DNA database search results, Forensic Sci. Int.: Genet. 5 (2011) 316-328.

[6] S. Cowen, P. Debenham, A. Dixon, S. Kutranov, J. Thomson, K. Way, An investigation of the robustness of the consensus method of interpreting low-template DNA profiles, Forensic Sci. Int.: Genet. 5 (2011) 400-406.

[7] C.D. Steele, M. Greenhalgh, D.J. Balding, Verifying likelihoods for low template DNA profiles using multiple replicates, Forensic Sci. Int.: Genet. 13 (2014) 82-89.

[8] K.S. Grisedale, A. van Daal, Comparison of STR profiling from low template DNA extracts with and without the consensus profiling method, Investigative Genetics 3 (2012) 14.

[9] B. Kokshoorn, B.J. Blankers, Response to Grisedale and van Daal: Comparison of STR profiling from low template DNA extracts with and without the consensus profiling method, Investigative Genetics 4 (2013) 1.

[10] K.S. Grisedale, A. van Daal, Comment on Kokshoorn, B, and Blankers, BJ 'Response to Grisedale, KS and van Daal, A: Comparison of STR profiling from low template DNA extracts with and without consensus profiling method', Investigative Genetics 4 (2013) 2.

[11] S. Gittelson, C.R. Steffen, M.D. Coble, Expected net gain of low-template DNA analyses, Data in Brief, submitted.

[12] C.R. Hill, D.L. Duewer, M.C. Kline, M.D. Coble, J.M. Butler, U.S. population data for 29 autosomal STR loci, Forensic Sci. Int.: Genet. 7 (2013) e82-e83.

[13] S. Gittelson, A. Biedermann, S. Bozza, F. Taroni, Decision analysis for the genotype designation in low-template-DNA profiles, Forensic Sci. Int.: Genet. 9 (2014) 118-133.

[14] J. von Neumann, O. Morgenstern, The Theory of Games and Economic Behavior, Princeton University Press, 2nd edition, Princeton, 1947.

[15] H. Raiffa, R. Schlaifer, Applied Statistical Decision Theory, Harvard University Press, Boston, 1961.

[16] R.H. Strotz, Cardinal Utility, The American Economic Review 43 (1953) 384-397.

[17] K.B. Korb, A.E. Nicholson, Bayesian Artificial Intelligence, Chapman \& Hall/CRC Press, Boca Raton FL, 2011 : pages 128 and 342.

[18] P. Gill, R. Puch-Solis, J.M. Curran, The low-template-DNA (stochastic) threshold - its determination relative to risk analysis for national DNA databases, Forensic Sci. Int.: Genet. 3 (2009) 104-111.

[19] A.A. Mitchell, J. Tamariz, K. O'Connell, N. Ducasse, Z. Budimlija, M. Prinz, T. Caragine, Validation of a DNA mixture statistics tool incorporating allelic drop-out and drop-in, Forensic Sci. Int.: Genet. 6 (2012) 749-761.

[20] S. Petricevic, J.P. Whitaker, J. Buckleton, S. Vintiner, J. Patel, P. Simon, H. Ferraby, W. Hermiz, A. Russell, Validation and development of interpretation guidelines for low copy number (LCN) DNA profiling in New Zealand using the AmpFISTER® SGM Plus ${ }^{\text {TM }}$ multiplex, Forensic Sci. Int.: Genet. 4 (2010) 305-310.

[21] Applied Biosystems Life Technologies. AmpFISTR®Identifiler® Plus PCR Amplification Kit User Guide. Publication Number 4440211, Revision F, Thermo Fisher Scientific, Inc., Carlsbad, 2015.

[22] Promega. PowerPlex® 16 HS System: Instructions for Use of Products DC2100 and DC2101. TMD022, Revised 2/16, Promega Corporation, Madison, 2015. 